\title{
Widespread Increased Diffusivity Reveals Early Cortical Degeneration in Huntington Disease
}

\author{
(D)F. Sampedro, (D) S. Martínez-Horta, (D). Perez-Perez, (D)A. Horta-Barba, (D). Martin-Lahoz, (D)A. Alonso-Solís, (D). Corripio,
} (D)B. Gomez-Anson, and (D). Kulisevsky

\begin{abstract}
BACKGROUND AND PURPOSE: Huntington disease is a devastating genetic neurodegenerative disorder for which no effective treatment is yet available. Although progressive striatal atrophy is its pathologic hallmark, concomitant cortical deterioration is assumed to occur, but it is poorly characterized. Our objective was to study the loss of cortical integrity and its association with clinical indicators throughout the course of the disease.
\end{abstract}

MATERIALS AND METHODS: Using a cohort of 39 patients with Huntington disease and 25 controls with available MR imaging (TIWI and DTI), we compared cortical atrophy and intracortical diffusivity across disease stages. Intracortical diffusivity is a DTI-derived metric that has recently been suggested to detect incipient neuronal death because water can diffuse more freely in cortical regions with reduced neural density.

RESULTS: We observed progressive thinning and increasing diffusivity within the cerebral cortex of patients with Huntington disease $(P<$ .05 , corrected for multiple comparisons). Most important, in the absence of pronounced atrophy, widespread increased diffusivity was already present in individuals with premanifest Huntington disease, correlating, in turn, with clinical and disease-specific progression markers.

CONCLUSIONS: Intracortical diffusivity may be more sensitive than cortical thinning for tracking early neurodegeneration in Huntington disease. Moreover, our findings provide further evidence of an early cortical compromise in Huntington disease, which contributes to our understanding of its clinical phenotype and could have important therapeutic implications.

ABBREVIATIONS: $C A G=$ cytosine-adenine-guanine; $C N$ = healthy controls; $C$ th $=$ cortical thickness; $D B S=$ disease burden score; earlyHD = early-symptomatic $\mathrm{HD} ; \mathrm{HD}=$ Huntington disease; $\mathrm{MD}=$ mean diffusivity; midadvHD = middle-advanced HD; preHD = premanifest HD; SDMT = Symbol Digit Modalities Test; $T$ MT = Trail-Making Test; UHDRS = Unified Huntington's Disease Rating Scale

$\mathrm{H}$ untington disease (HD) is a fatal neurologic disorder with no effective treatment currently available. Inherited in an autosomal-dominant pattern, it is the most prevalent monogenic neurodegenerative disease. A cytosine-adenine-guanine (CAG) triplet repeat expansion in the huntingtin gene (HTT) drives a pathologic brain aggregation of mutant huntingtin protein, which promotes neuronal cell injury and death. Typically around the

Received January 29, 2019; accepted after revision July 7.

From the Movement Disorders Unit (F.S., S.M.-H., J.P.-P., A.H.-B., J.M.-L., J.K.), Neurology Department; Neuroradiology, Radiology Department (B.G.-A.); and Psychiatry Department (I.C.), Hospital de la Santa Creu i Sant Pau, Barcelona, Spain; Biomedical Research Institute (F.S., S.M.-H., J.P.-P., A.H.-B., J.M.-L., A.A.-S., I.C., J.K.), Barcelona, Spain; Centro de Investigación en Red-Enfermedades Neurodegenerativas (F.S.,

S.M.-H., J.P.-P., A.H.-B., J.M.-L., J.K.), Madrid, Spain; Universitat Autónoma de Barcelona (S.M.-H., J.P.-P., J.M.-L., B.G.-A., J.K.), Barcelona, Spain; European Huntington's Disease Network (S.M.-H., J.P.-P., A.H.-B., J.K.), Ulm, Germany; and Centro de Investigación Biomédica en Red-Salud Mental (I.C.), Madrid, Spain.

This study was partially funded by Fondo de Investigaciones Sanitarias (FIS) of the Instituto de Salud Carlos III (ISCIII) and Fondo Europeo de Desarrollo Regional (FEDER), Grant No. PI17/001885. fourth decade of life, HD gene mutation carriers experience progressive motor, cognitive, and neuropsychiatric alterations, resulting in a devastating loss of functional independence. ${ }^{1} \mathrm{Al}-$ though the pathologic hallmark of HD is a massive loss of the medium spiny neurons of the striatum, the current view of this disorder is that diffuse cortical and subcortical neurodegeneration underlies the clinical picture. ${ }^{2}$

Characterizing early brain degeneration in HD may prove critical to designing and monitoring novel therapeutic strategies to prevent or delay its clinical onset. Neuroimaging indicators hold great potential in this setting, but their sensitivity and interpretation need further assessment. In this vein, the study of individuals

Please address correspondence to Jaime Kulisevsky, MD, PhD, Movement Disorders Unit, Neurology Department, Hospital de la Santa Creu i Sant Pau, Mas Casanovas 90-08041 Barcelona, Spain; e-mail: jkulisevsky@santpau.cat

-- Indicates open access to non-subscribers at www.ajnr.org

三 Indicates article with supplemental on-line tables.

http://dx.doi.org/10.3174/ajnr.A6168 
with genetically confirmed-but-premanifest HD (preHD) offers a unique opportunity to evaluate the performance of imaging indicators in identifying early neurodegeneration. In contrast to preclinical subjects at risk for other neurodegenerative disorders, such as sporadic Alzheimer disease or Parkinson disease, individuals with premanifest HD will inevitably develop clinical symptoms in the upcoming years. Imaging indicators that best identify cortical and subcortical deterioration in subjects with premanifest HD would therefore be good candidates for use in clinical trials to noninvasively monitor early neurodegeneration.

In this context, MR imaging indicators are the best-suited approach to monitor disease progression because of their high-resolution and radiation-free acquisition. However, detecting subtle signs of early cortical deterioration with this technique may be challenging due to a number of technical and conceptual limitations of the most commonly used MR imaging indicators. Atrophy assessment via voxel-based morphometry (VBM) or cortical thickness (Cth), for example, is highly specific, but it may not be sufficiently sensitive to detect early neural loss. ${ }^{3}$ Second, white matter integrity estimated from diffusion tensor imaging does not directly assess the loss of cortical neurons, which is probably the main mechanism underlying cognitive and neuropsychiatric symptoms in neurodegenerative diseases. Third, clinical interpretation of functional MR imaging measures such as resting-state connectivity is challenging due to the complex nature of imaging indicators and the presence of compensatory mechanisms.

In view of these limitations, imaging markers capable of detecting early loss of neural tissue within the cerebral cortex need to be proposed and validated for the study of neurodegeneration. It was recently suggested that an increase in intracortical mean diffusivity (MD) of water molecules reflects early cortical compromise. ${ }^{4,5}$ Conceptually, an incipient degeneration of cortical neurons that do not yet translate into cortical thinning may be detected through a regional increase of water mobility due to the recent cell death. MD data can be derived from DTI scans, and in contrast to other DTI-derived metrics such as fractional anisotropy, regional MD is an adequate indicator of gray matter integrity due to its isotropic structure. ${ }^{4}$ Moreover, this measure has recently shown higher effect sizes and increased sensitivity in identifying cortical degeneration in other neurodegenerative diseases. $^{5}$

In this work, we characterized intracortical MD and atrophy changes across HD stages. Our main objective was to investigate whether MD abnormalities precede atrophy detection. Our secondary objective was to study the possible associations between these imaging alterations and the patients' motor, cognitive, and neuropsychiatric statuses.

\section{MATERIALS AND METHODS}

\section{Participants and Clinical Assessments}

We included 39 HD gene-mutation carriers (CAG $\geq 39$ ) from the outpatient clinic of the Movement Disorders Unit at Hospital de la Santa Creu i Sant Pau (Barcelona, Spain). Within this HD group, participants were classified as having preHD, early-symptomatic HD (earlyHD), or middle-advanced HD (midadvHD) according to previously defined criteria. ${ }^{6-8}$ Individuals with a Unified Huntington's Disease Rating Scale (UHDRS) Total
Motor Score of $<5$ and a diagnostic confidence level of $<3$ were classified as having preHD. Patients showing a Total Motor Score of $\geq 5$, a diagnostic confidence level of 4 , and a Total Functional Capacity Scale score between 11 and 13 were classified as having earlyHD, and those showing a Total Functional Capacity Scale score of $<11$ were combined to form a midadvHD group. We also included 25 healthy controls $(\mathrm{CN})$ who had no family history of movement or psychiatric disorders. This $\mathrm{CN}$ group had an age distribution similar to that of the preHD group because the imaging comparison between $\mathrm{CN}$ and preHD would be key to evaluating the sensitivity of early markers of neurodegeneration.

We recorded demographic, motor, cognitive, neuropsychiatric, and disease-specific indicators for all HD participants. We used the disease burden score (DBS), defined as age $\times$ (CAG 35.5) as an index of the burden of pathology due to lifetime exposure to mutant huntingtin. ${ }^{9}$ In subjects with premanifest $\mathrm{HD}$, the estimated years to clinical onset can be obtained by the formula described in Langbehn et al. ${ }^{10}$ Functional capacity, motor symptoms, and global cognitive status were recorded using the UHDRS. We also addressed the severity of apathy, the most prevalent and characteristic neuropsychiatric feature of HD. ${ }^{6}$ Last, we administered the UHDRS cognitive subtest with additional cognitive tasks. We recorded the following set of cognitive indicators, known to be sensitive to HD progression: the Symbol Digit Modalities Test (SDMT), the Stroop Task, the Trail-Making Test (TMT), the F-A-S verbal fluency test, and semantic fluency. ${ }^{11}$

\section{Neuroimaging Acquisition and Methods}

Volumetric MR imaging and DTI were available for all participants. 3D-T1 images were acquired on a 3T Achieva scanner (Philips Healthcare, Best, the Netherlands) using an MPRAGE sequence $\left(\mathrm{TR} / \mathrm{TE}=6.74 / 3.14 \mathrm{~ms}\right.$, flip angle $=8^{\circ}, \mathrm{FOV}=23 \mathrm{~cm}$, matrix $=256 \times 256$, and slice thickness $=1 \mathrm{~mm}$ ). DTI scans were also obtained using the following acquisition parameters: $\mathrm{TR}=$ $10,433 \mathrm{~ms}, \mathrm{TE}=57 \mathrm{~ms}$, slice thickness $=2 \mathrm{~mm}$, flip angle $=90^{\circ}$, 15 directions, $\mathrm{b}$ factor $=800$.

For each participant, we quantified cortical thickness and intracortical diffusivity. Cortical thickness analysis was performed using the FreeSurfer 6.0 software package (http://surfer. nmr.mgh.harvard.edu). The specific methods used for cortical reconstruction of volumetric images have been fully described elsewhere. ${ }^{12}$ Briefly, optimized surface-deformation models following intensity gradients accurately identify white matter and gray matter boundaries in the cerebral cortex, from which cortical thickness is computed at each vertex. On visual inspection, no major surface reconstruction errors were observed in our sample. Mean diffusivity maps were computed from DTI scans and aligned to the associated T1-weighted image using the FreeSurfer module Tracula (https://surfer.nmr.mgh.harvard.edu/fswiki/ FsTutorial/Tracula). ${ }^{13}$ To study differences in MD across groups, we first used the FreeSurfer's partial volume correction (PVC) tools to account for a possible volume fraction effect (i.e. concomitant atrophy). We then sampled intracortical MD values half-way between the white and pial surfaces of the cortical ribbon, thereby obtaining surface-based vertex-wise MD data along the cerebral cortex. By definition, Cth and intracortical MD can only be measured in cortical regions. In subcortical regions, we also computed 
Clinical and demographic information across groups ${ }^{a}$

\begin{tabular}{|c|c|c|c|c|c|c|}
\hline & $\mathrm{CN}$ & HD & PreHD & EarlyHD & MidadvHD & $P$ Value \\
\hline No. & 25 & 39 & 15 & 16 & 8 & \\
\hline Age (yr) & $38.6 \pm 9.4$ & $47.8 \pm 13.6$ & $37.8 \pm 10.9$ & $55.0 \pm 11.8$ & $52.3 \pm 10.4$ & $.0044^{\mathrm{b}} .81,,^{\mathrm{c}}<.001,{ }^{\mathrm{d}} .57^{\mathrm{e}}$ \\
\hline Female sex (\%) & $36 \%$ & $69 \%$ & $80 \%$ & $56 \%$ & $75 \%$ & $.01, .01, .16, .37$ \\
\hline Education (yr) & $12.1 \pm 1.9$ & $13.4 \pm 5.7$ & $16.4 \pm 3.2$ & $11.1 \pm 5.2$ & $12.5 \pm 6.5$ & $.24,<.001, .002, .61$ \\
\hline CAG length & NA & $43.1 \pm 2.9$ & $43.1 \pm 2.9$ & $42.6 \pm 2.9$ & $43.8 \pm 2.9$ & $\mathrm{NA}, \mathrm{NA}, .59, .31$ \\
\hline DBS $^{f}$ & NA & $339.6 \pm 105.3$ & $273.3 \pm 108.9$ & $364.5 \pm 78.2$ & $414.4 \pm 78.1$ & NA, NA, .01, .15 \\
\hline Estimated yr to onset ${ }^{g}$ & NA & NA & $15.8 \pm 7.8$ & NA & NA & \\
\hline TFC & NA & $11.3 \pm 3.0$ & $12.9 \pm 0.2$ & $12.1 \pm 0.9$ & $6.4 \pm 3.5$ & NA, NA, .004, <.001, \\
\hline UHDRS-TMS & NA & $20.6 \pm 20.7$ & $1.4 \pm 1.5$ & $23.6 \pm 14.2$ & $50.5 \pm 8.3$ & $\mathrm{NA}, \mathrm{NA},<.001,<.001$ \\
\hline UHDRS-Cogscore & NA & $220.6 \pm 112.8$ & $323.9 \pm 48.4$ & $189.4 \pm 66.1$ & $65.8 \pm 60.2$ & $\mathrm{NA}, \mathrm{NA},<.001,<.001$ \\
\hline Apathy score ${ }^{\mathrm{h}}$ & NA & $4.9 \pm 5.3$ & $2.5 \pm 3.7$ & $4.6 \pm 5.4$ & $9.8 \pm 4.7$ & $\mathrm{NA}, \mathrm{NA},=.2, .03$ \\
\hline FAS & NA & $28.5 \pm 17.3$ & $43.6 \pm 12.4$ & $22.7 \pm 12.3$ & $9.7 \pm 6.4$ & NA, NA, $<.001, .01$ \\
\hline SDMT & NA & $35.1 \pm 20.1$ & $54.7 \pm 8.4$ & $27.7 \pm 12.9$ & $9.8 \pm 8.1$ & $\mathrm{NA}, \mathrm{NA},<.001, .001$ \\
\hline Semantic fluency & NA & $16.4 \pm 7.0$ & $23.0 \pm 4.2$ & $14.3 \pm 3.5$ & $7.1 \pm 3.3$ & $\mathrm{NA}, \mathrm{NA},<.001, .001$ \\
\hline Stroop Color & NA & $51.6 \pm 28.9$ & $75.5 \pm 20.6$ & $44.3 \pm 17.8$ & $16.8 \pm 19.5$ & $\mathrm{NA}, \mathrm{NA},<.001, .01$ \\
\hline Stroop Word & NA & $71.9 \pm 34.5$ & $99.2 \pm 19.1$ & $68.1 \pm 21.7$ & $22.0 \pm 22.4$ & $\mathrm{NA}, \mathrm{NA},<.001, .001$ \\
\hline TMT (parts B-A) & NA & $-4.6 \pm 8.0$ & $0.3 \pm 0.7$ & $-3.6 \pm 5.1$ & $-17.6 \pm 8.5$ & NA, NA, .008, .004 \\
\hline TMT cognitive flexibility & NA & $1.5 \pm 1.4$ & $1.1 \pm 1.1$ & $2.0 \pm 1.4$ & $1.0 \pm 1.4$ & NA, NA, .07, .15 \\
\hline
\end{tabular}

Note:-NA indicates not available; TFC, Total Functional Capacity; TMS, Total Motor Score; Cogscore, Cognitive Score; FAS, F-A-S test of verbal fluency.

${ }^{a}$ Values are expressed as mean \pm standard deviation.

${ }^{\mathrm{b}} \mathrm{CN}$ vs HD.

${ }^{c} \mathrm{CN}$ vs preHD.

${ }^{\mathrm{d}}$ PreHD vs earlyHD.

e EarlyHD vs midadvHD.

${ }^{\mathrm{f}} \mathrm{DBS}=9$.

8 Derived from 10

${ }^{\mathrm{h}}$ Derived from 23

volume information and average MD values in common subcortical structures (caudate, putamen, accumbens, pallidum, amygdala, thalamus, and hippocampus).

\section{Statistical Analyses}

We compared clinical, sociodemographic, and scalar subcortical volumetric and MD data across groups using 2-sample $t$ test analysis for continuous variables and the $\chi^{2}$ fest for categoric variables. Differences were considered significant using a probability value $<.05$.

Cortical vertex-wise measures (Cth and MD) were first smoothed using a Gaussian kernel of 10-mm full width at half maximum to increase the signal-to-noise ratio. A generalized linear model was then performed to compare these measures across groups, using age, sex, and education as covariates of no interest. Clusters surviving $P<.05$ and family-wise error correction for multiple comparison by a Monte Carlo simulation with 10,000 repeats were considered significant.

Finally, aiming to investigate the clinical translation of the imaging findings, we computed mean atrophy and MD values at the identified cortical clusters and subcortical regions showing significant differences across groups. Using Pearson correlation coefficients, we then studied the association of these imaging measures with clinical indicators within an exploratory analysis, for which a $P<.05$ was considered significant.

\section{RESULTS}

The Table summarizes the demographic, clinical, and neuropsychological characteristics of all participants.

A clinical decay in motor, cognitive, and neuropsychiatric indicators was observed across HD stages. Patients with premanifest $\mathrm{HD}$ were younger than those with manifest $\mathrm{HD}$, but differences in genetic burden across HD groups were not significant.
The Figure shows cortical thickness and intracortical MD differences across HD groups with respect to the $\mathrm{CN}$ group.

Progressive cortical thinning and increasing diffusivity were observed across HD stages. However, MD alterations were more pronounced and more extensive than atrophic differences in all HD groups. In the preHD group, whereas only 3 relatively small atrophic clusters were shown (left caudal middle frontal, left inferior-parietal, and right inferior-temporal), diffusivity was increased in precentral, rostral middle and superior-frontal, entorhinal, posterior cingulate/precuneus, superior-temporal, insula, bankssts region, and inferior-parietal and postcentral regions. Patients with earlyHD showed widespread cortical thinning and increased MD. The following regions showed cortical thinning with the highest significance $(P<.005$ family-wise error): superiorfrontal/temporal-parietal, inferior-temporal/parietal, postcentral, cingulate isthmus, and supramarginal gyrus. In contrast, the most significant increases in MD were found in caudal middle frontal, orbitofrontal, precentral, pericalcarine, cuneus, precuneus, and superior-temporal/-parietal regions. In particular, MD increases in the absence of Cth differences in patients with earlyHD were observed in the following regions: superior-frontal gyri, occipital and entorhinal cortices, and temporal poles. Regarding patients with midadvHD, because atrophy and increased MD were widespread throughout the cerebral cortex, no further explorations were performed.

On-line Table 1 shows volumetric and MD information in common subcortical structures across groups.

Progressive atrophy and increasing diffusivity were observed in most subcortical structures across $\mathrm{CN}$, preHD, earlyHD, and midadvHD groups. While these changes were most pronounced in the striatum, other important structures such as the thalamus, hippocampus, and amygdala were also involved. Similar signifi- 


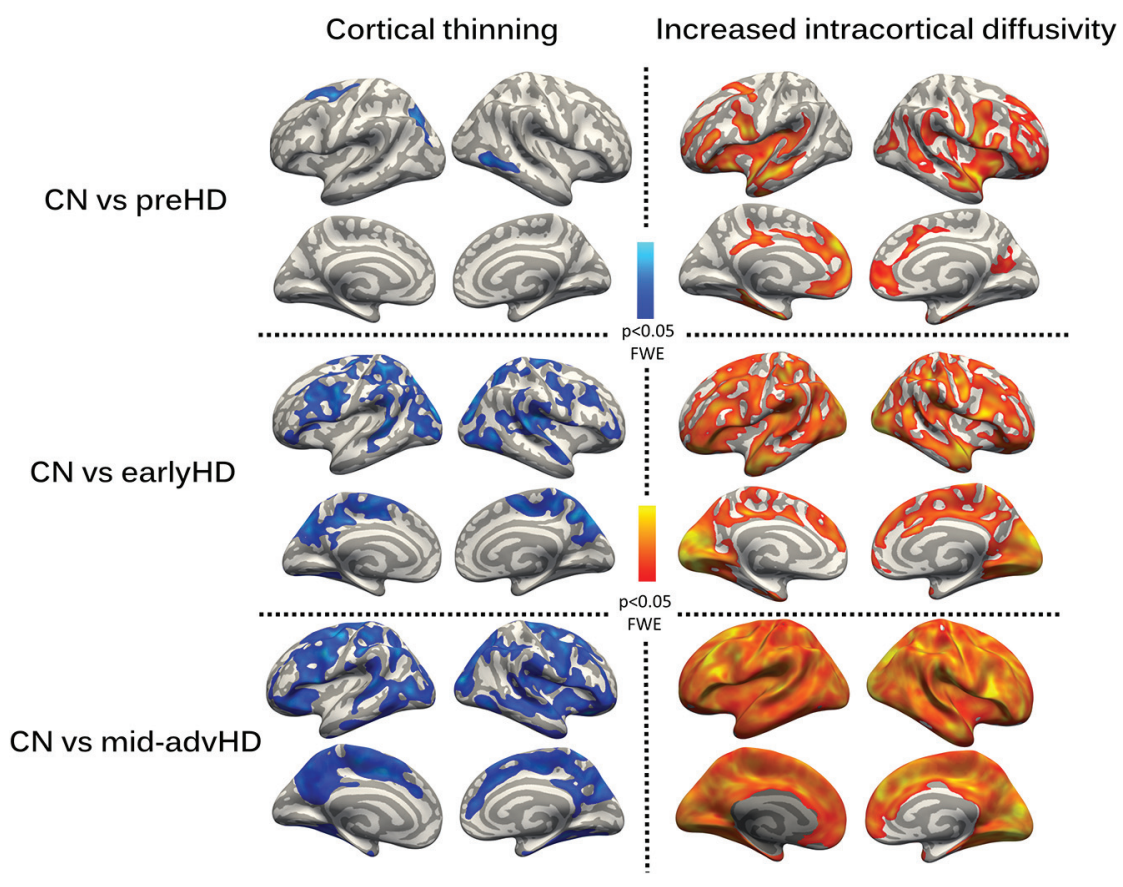

FIGURE. Differences in intracortical MD and cortical thickness across HD stages with respect to $\mathrm{CN}$, using age, sex, and education as covariates of no interest $(P<.05$ family-wise error).

within the cerebral cortex. We found that clinical deterioration in this population concurred with progressive cortical thinning and increases in diffusivity. Additionally, as expected, concomitant subcortical impairment along disease stages was also revealed by both imaging modalities.

Of particular note among our findings was the widespread increase in intracortical diffusivity that was already evidenced in individuals with premanifest HD with respect to the control group, even in regions with preserved cortical thickness. This imaging indicator has been suggested to reflect an incipient neural loss within the cerebral cortex. ${ }^{4}$ Most important, MD values at the identified cortical regions correlated with inherent markers of disease progression (DBS, estimated years to onset) and with motor and cognitive measures. From an imaging perspective, because cortical thinning was not detected in

cant differences were found across groups in terms of subcortical volumetric and MD measures. Patients with preHD showed lower subcortical volumes than $\mathrm{CN}$ in all the regions considered. They also showed increased MD in all these regions except in the pallidum, left amygdala, right accumbens, and right thalamus. Patients with earlyHD showed lower subcortical volumes than those with preHD except for the left accumbens, bilateral amygdala, and bilateral thalamus and hippocampus; and subcortical diffusivity was increased in the striatum and left thalamus.

Finally, On-line Table 2 illustrates a set of exploratory correlations between imaging indicators and clinical information within the preHD and earlyHD groups. $\mathrm{CN}$ and midadvHD were not considered in this analysis due to missing information and massive atrophic and clinical deterioration, respectively. In Online Table 3, these correlations were controlled for caudate atrophy to identify independent contributions of cortical degeneration to clinical indicators.

Diffusivity abnormalities showed good correlations with preclinical indicators of disease progression (DBS, estimated years to onset) and also with motor, cognitive, and neuropsychiatric status. Volumetric and Cth degeneration showed a much lower number of significant associations with clinical indicators, especially within the preHD group. Topographically, clinical-imaging associations were not restricted to frontostriatal territories. Even after controlling for caudate atrophy, significant clinical-imaging correlations were found in a large number of frontotemporoparieto-occipital clusters and extrastriatal subcortical regions (eg, thalamus, hippocampus).

\section{DISCUSSION}

In this work, we characterized cortical microstructural changes across Huntington disease stages using intracortical diffusivity, a promising image-derived indicator of early neurodegeneration most of these regions, these results suggest that intracortical MD may outperform Cth at tracking early cortical neurodegeneration in HD. In addition, because both subcortical MD and volumetric information appropriately characterized disease progression in our sample, MD indicators show the potential for use in clinical trials. Furthermore, the fact that we observed a substantial cortical compromise in patients with earlyHD strongly suggests that future neuroimaging studies should look beyond subcortical alterations because analyses only restricted to those regions (such as ${ }^{14-16}$ ) may overlook some important contributions of cortical degeneration in the HD phenotype.

From a clinical point of view, identifying early cortical and subcortical degeneration beyond frontostriatal territories might contribute to a better understanding of clinical manifestations in HD. We observed an association between cognitive performance (UHDRS-Cognitive Score, Stroop, and SDMT) and imaging indicators in key brain regions such as the entorhinal cortex, precuneus, and hippocampus. Most important, the compromise of these key brain regions was previously evidenced in the neuroimaging analyses. These results suggest that even in early disease stages, the ongoing neurodegeneration in HD impacts important brain circuitry beyond frontostriatal areas. In this vein, this early cortical microstructural compromise probably leads to functional connectivity or white matter abnormalities, which have been previously related to clinical manifestations in HD. ${ }^{17-19}$ Taken together, our findings reinforce the need to consider HD as a wholebrain neurodegenerative disease even from its early stages, as evidenced by the diffuse cortical damage observed in individuals with premanifest HD. Most interesting, early cortical deterioration may underlie the onset of cognitive and neuropsychiatric symptoms in HD.

From a biologic perspective, a first point to note is that we did not find imaging evidence of neuroinflammation in HD because 
no brain region showed a decrease in cortical diffusivity. ${ }^{20,21} \mathrm{Nev}-$ ertheless, the specific pathologic mechanisms responsible for early cortical damage in HD need further assessment. In this context, PET imaging using mutant huntingtin tracers could help to disentangle whether this abnormal protein aggregates and damages the cerebral cortex or whether cortical deterioration is driven by a parallel and partially independent pathologic pathway. ${ }^{22}$

The main strength of this study is the use of a multimodal surface-based imaging approach that includes PVC-MD assessment, a promising indicator of early cortical degeneration. Additionally, we were able to characterize the clinical-imaging continuum of a well-characterized HD sample.

The study has 2 main limitations, the first of which is the relatively low number of patients and a control group that lacked a detailed cognitive assessment. The second limitation is that cross-sectionality and exploratory clinical-imaging correlations could hinder the interpretation of our results.

\section{CONCLUSIONS}

The early widespread increase in diffusivity within the cerebral cortex of patients with HD correlated with clinical indicators of disease progression. Our results provide further evidence of concomitant corticostriatal degeneration in HD, which contributes to our understanding of its phenotype and may have clinical and therapeutic implications.

\section{ACKNOWLEDGMENTS}

The authors wish to thank the study team and all the study participants and their families.

Disclosures: Jesus Perez-Perez-RELATED: Grant: Fondo de Investigaciones Sanitarias de España Spanish Government, Carlos III Health Institute, Comments: This study was partially funded by Fondo de Investigaciones Sanitarias de España Spanish Government grant No. PI17/001885*; UNRELATED: Grants/Grants Pending: Fondo de Investigaciones Sanitarias de España Spanish Government, Carlos III Health Institute, Comments: I am the Principal Investigator of grant No. PI17/001885.* *Money paid to the institution.

\section{REFERENCES}

1. Walker FO. Huntington's disease. Lancet 2007;369:218-28 CrossRef Medline

2. McColgan P, Tabrizi SJ. Huntington's disease: a clinical review. Eur J Neurol 2018;25:24-34 CrossRef Medline

3. Haller S, Xekardaki A, Delaloye C, et al. Combined analysis of grey matter voxel-based morphometry and white matter tract-based spatial statistics in late-life bipolar disorder. J Psychiatry Neurosci 2011;36:391-401 CrossRef Medline

4. Weston PSJ, Simpson IJA, Ryan NS, et al. Diffusion imaging changes in grey matter in Alzheimer's disease: a potential marker of early neurodegeneration. Alzheimers Res Ther 2015;7:47 CrossRef Medline

5. Illán-Gala I, Montal V, Borrego-Écija S, et al; Catalan Frontotemporal Dementia Initiative (CATFI) and the Frontotemporal Lobar Degeneration Neuroimaging Initiative (FTLDNI). Cortical microstructure in the behavioural variant of frontotemporal dementia: looking beyond atrophy. Brain 2019;142:1121-33 CrossRef Medline
6. Martinez-Horta S, Perez-Perez J, van Duijn E, et al; Spanish REGISTRY investigators of the European Huntington's Disease Network. Neuropsychiatric symptoms are very common in premanifest and early stage Huntington's disease. Parkinsonism Relat Disord 2016;25:58-64 CrossRef Medline

7. Tabrizi SJ, Langbehn DR, Leavitt BR, et al; TRACK-HD investigators. Biological and clinical manifestations of Huntington's disease in the longitudinal TRACK-HD study: cross-sectional analysis of baseline data. Lancet Neurol 2009;8:791-801 CrossRef Medline

8. Shoulson I, Fahn S. Huntington disease: clinical care and evaluation. Neurology 1979;29:1-3 CrossRef Medline

9. Penney JB Jr, Vonsattel JP, MacDonald ME, et al. CAG repeat number governs the development rate of pathology in Huntington's disease. Ann Neurol 1997;41:689-92 CrossRef Medline

10. Langbehn DR, Hayden M, Paulsen JS; PREDICT-HD Investigators of the Huntington Study Group. CAG-repeat length and the age of onset in Huntington disease (HD): a review and validation study of statistical approaches. Am J Med Genet B Neuropsychiatr Genet 2010; 153B:397-408 CrossRef Medline

11. Terwindt PW, Hubers AA, Giltay EJ, et al. Screening for cognitive dysfunction in Huntington's disease with the clock drawing test. Int J Geriatr Psychiatry 2016;31:1013-20 CrossRef Medline

12. Fischl B, Dale AM. Measuring the thickness of the human cerebral cortex from magnetic resonance images. Proc Natl Acad Sci U S A 2000;97:11050-55 CrossRef Medline

13. Yendiki A, Panneck P, Srinivasan P, et al. Automated probabilistic reconstruction of white-matter pathways in health and disease using an atlas of the underlying anatomy. Front Neuroinform 2011;5:23 CrossRef Medline

14. Niccolini F, Pagano G, Fusar-Poli P, et al. Striatal molecular alterations in HD gene carriers: a systematic review and meta-analysis of PET studies. J Neurol Neurosurg Psychiatry 2018;89:185-96 CrossRef Medline

15. Hobbs NZ, Barnes J, Frost C, et al. Onset and progression of pathologic atrophy in Huntington disease: a longitudinal MR imaging study. AJNR Am J Neuroradiol 2010;31:1036-41 CrossRef Medline

16. Aylward EH, Rosenblatt A, Field $\mathrm{K}$, et al. Caudate volume as an outcome measure in clinical trials for Huntington's disease: a pilot study. Brain Res Bull 2003;62:137-41 CrossRef Medline

17. Gregory S, Scahill RI, Seunarine KK, et al. Neuropsychiatry and white matter microstructure in Huntington's disease. J Huntingtons Dis 2015;4:239-49 CrossRef Medline

18. Wolf RC, Sambataro F, Vasic N, et al. Default-mode network changes in preclinical Huntington's disease. Exp Neurol 2012;237: 191-98 CrossRef Medline

19. Klöppel S, Stonnington CM, Petrovic P, et al. Irritability in pre-clinical Huntington's disease. Neuropsychologia 2010;48:549-57 CrossRef Medline

20. Montal V, Vilaplana E, Alcolea D, et al. Cortical microstructural changes along the Alzheimer's disease continuum. Alzheimers Dement 2018;14:340-51 CrossRef Medline

21. Gispert JD, Suárez-Calvet M, Monté GC, et al. Cerebrospinal fluid sTREM2 levels are associated with gray matter volume increases and reduced diffusivity in early Alzheimer's disease. Alzheimers Dement 2016;12:1259-72 CrossRef Medline

22. Simmons DA, James ML, Belichenko NP, et al. TSPO-PET imaging using $[18 \mathrm{~F}] \mathrm{PBR} 06$ is a potential translatable biomarker for treatment response in Huntington's disease: preclinical evidence with the p75NTR ligand LM11A-31. Hum Mol Genet 2018;27:2893-912 CrossRef Medline 